\title{
IGCC: uma alternativa para a utilização de carvão mineral brasileiro
}

\author{
João Mota Neto* \\ Diogo Kaminski*
}

\begin{abstract}
RESUMO - A expectativa da redução das reservas de fontes não renováveis, bem como a crescente preocupação com a preservação ambiental, vêm incentivando a substituição desses recursos e tecnologias convencionais na geração de energia elétrica por alternativas de maior desempenho e/ou fontes renováveis. Nesse âmbito, a gaseificação do carvão mineral está ganhando espaço devido à evoluções tecnológicas, que possibilitam a geração de energia elétrica de maneira limpa e com maior eficiência quando comparada com as consolidadas usinas termelétricas. A partir destas premissas, este trabalho abordará o crescimento da demanda energética nacional, uma análise dos custos de implantação da tecnologia IGCC e um comparativo econômico com outros meios de geração limpa.
\end{abstract}

Palavras-chave: Geração de energia elétrica. Carvão mineral. IGCC.

\section{INTRODUÇÃO}

Os países em crescimento econômico possuem em comum a necessidade de recursos energéticos como base para incremento do setor produtivo. $\mathrm{O}$ desenvolvimento sustentável também é fator relevante na análise dessa expansão, minimizando o impacto ambiental que o homem exerce sobre o planeta.

Entre os recursos energéticos não renováveis no mundo, o carvão mineral ocupa a primeira colocação em abundância e perspectiva de vida útil, a qual está estimada em 200 anos, destacando-se como fonte principal quando comparado ao petróleo e o gás natural, os quais apresentam vida útil de 40 e 65 anos, respectivamente. Na composição da matriz energética global, o carvão está posicionado abaixo do petróleo, contudo na geração de energia elétrica é o principal recurso mundial.

Conforme consta no balanço energético de 2010 do Ministério de Minas e Energia, a produção de carvão mineral encontra-se localizada na região sul do Brasil, onde o estado do Rio Grande do Sul retém 3.094.000 toneladas, Santa Catarina 2.522.000 e Paraná 93.000. A projeção da participação do carvão mineral na matriz energética nacional, em 2023, é de 5,35\%.

Entretanto, o principal obstáculo para aumentar a participação do carvão na matriz energética nacional é o fato de que atualmente a maioria dos países faz a geração de energia através da queima direta desse combustível. Esse processo gera elevada quantidade de gases * Mestre em Engenharia Mecânica pela Universidade Federal do Rio Grande do Sul. É professor da Faculdade SATC. Endereço eletrônico: joão.neto@satc.edu.br.

** Engenheiro Mecânico pela SATC. É pesquisador do CNPq. Endereço eletrônico: diogo.kaminski@satc.edu.br. 
poluentes, os quais contribuem para o aquecimento global. O carvão ainda será largamente utilizado para geração de energia elétrica, haja vista seu baixo preço, alta disponibilidade e distribuição desse combustível.

Visando aumentar a utilização do carvão mineral para geração de eletricidade em conformidade com as normas ambientais em vigência, a comunidade científica está desenvolvendo novas tecnologias. Dentre essas, se destaca o IGCC (Integrated Gasification Combined Cycle), que se apresenta com maior possibilidade de implantação em escala industrial devido aos seus custos tecnoeconômicos.

\section{PANORAMA DO CONSUMO ENERGÉTICO NACIONAL}

Segundo dados das Centrais Elétricas Brasileiras S. A. (ELETROBRAS), o consumo de energia elétrica no Brasil cresceu mais de 1.100\% nos últimos 40 anos, a uma taxa média de 6,75\% ao ano. O crescimento mais expressivo ocorreu na década de 70 , período denominado "Milagre econômico", quando o consumo energético cresceu a taxas superiores de $10 \%$ ao ano. Sendo principalmente as hidrelétricas responsáveis por atender o crescimento na demanda nacional, é necessário diversificar a matriz energética a fim de garantir estabilidade no fornecimento e evitando apagões.

O consumo de energia elétrica em 2010, segundo a EPE (Empresa de Pesquisa Energética), foi de 419.010 GWh, valor este que está um pouco acima das previsões, porém deve se levar em conta variações na demanda anual de acordo com o crescimento e o desenvolvimento do país. O fato é que a demanda cresce cada vez mais com o passar dos anos, ressaltando a importância da implantação de novas unidades e diversificação da matriz energética nacional.

Na Tabela 1 apresenta-se a projeção da demanda energética nacional para 2011 a 2015, considerando dois cenários para crescimento médio anual da economia brasileira, de 4,87\% para um baixo desempenho e 5,04\% num cenário econômico favorável (REGO, 2004). Para este cálculo considerou-se todas as modalidades de consumidores, sendo estes: industriais, residenciais, comerciais, rurais, iluminação e serviço público.

TABELA 1 - PROJEÇÃO NO AUMENTO DE DEMANDA

\begin{tabular}{l|r|r|r|r|c}
\hline & $\mathbf{2 0 1 1}$ & $\mathbf{2 0 1 2}$ & $\mathbf{2 0 1 3}$ & $\mathbf{2 0 1 4}$ & $\mathbf{2 0 1 5}$ \\
\hline Demanda baixa (GWh) & 438.610 & 460.443 & 478.002 & 503.783 & 531.798 \\
Demanda alta (GWh) & 444.263 & 467.118 & 485.776 & 513.017 & 542.525 \\
\hline
\end{tabular}

FONTE: REGO (2004).

\section{DESCRIÇÃO DO PROCESSO IGCC}

Define-se IGCC (Integrated Gasification Combined Cycle - Ciclo Combinado com Gaseifi- 
cação Integrada) como um processo para geração de energia elétrica através da gaseificação do carvão, por meio de turbinas a gás e a vapor, sendo que a novidade reside na integração dessas turbinas para geração de energia elétrica.

O IGCC vem se desenvolvendo a partir da combinação de duas tecnologias: a geração de energia em ciclo combinado e a gaseificação de combustíveis sólidos, conforme ilustra a Figura 1. A geração em ciclo combinado se destaca por sua alta eficiência: enquanto a eficiência nominal de uma termelétrica a gás em ciclo simples atinge de 33\% a 42\%, na geração em ciclo combinado se alcançam eficiências nominais de $59 \%$, se referindo ao poder calorífico inferior (DOLEZAL, 2001).

FIGURA 1 - CICLO COMBINADO COM GASEIFICAÇÃO INTEGRADA

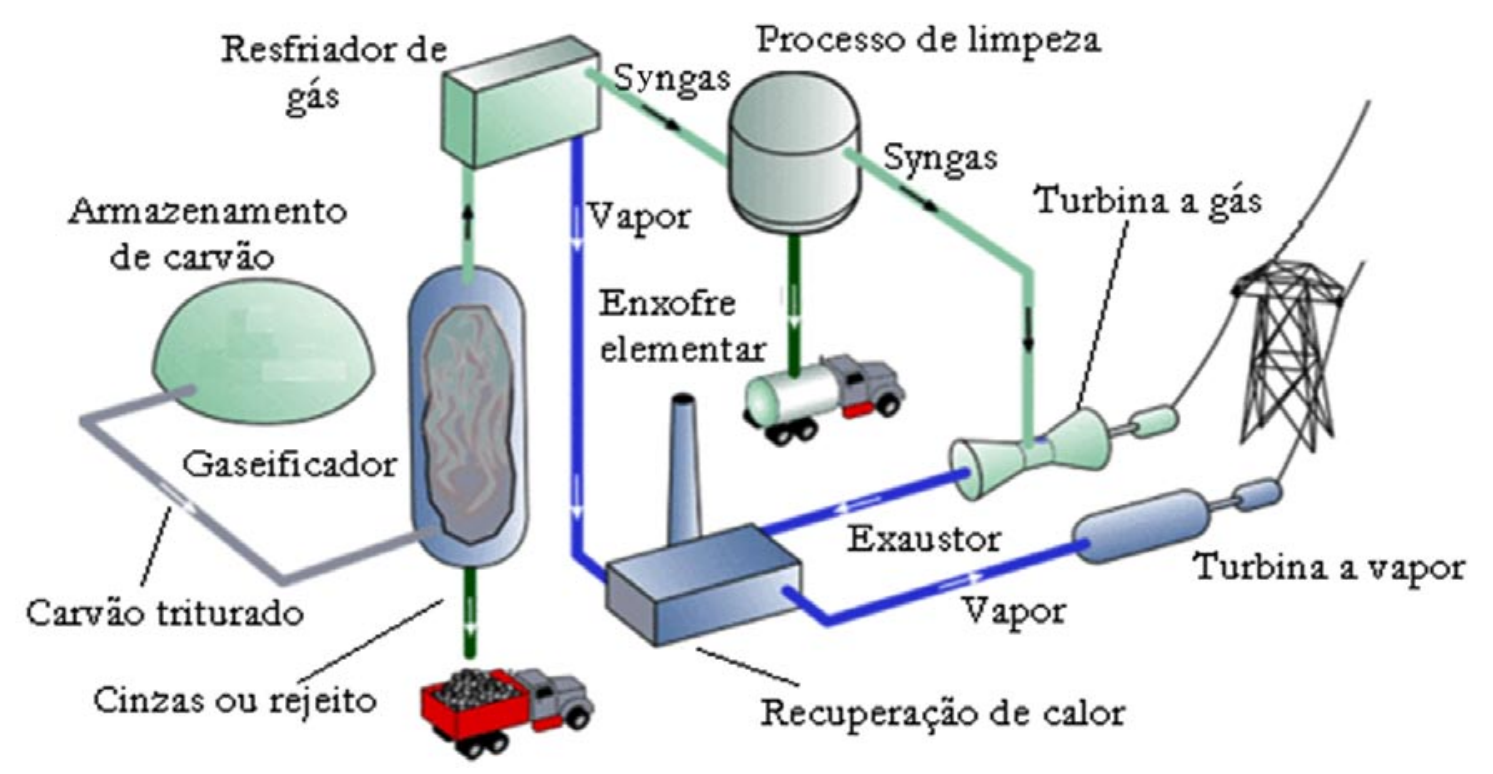

FONTE: World Coal Association (2011).

\subsection{CUSTOS ENVOLVIDOS NA GERAÇÃO DE ENERGIA ELÉTRICA ATRAVÉS DO IGCC}

Neste item será determinado o custo da geração de energia elétrica produzida através da tecnologia IGCC, a qual utiliza carvão mineral como combustível. Ressalta-se que nos custos apresentados não está incluído o sistema de captura de carbono. Deste modo, são analisadas as plantas que foram concebidas conforme a estrutura básica apresentada na Figura 1 e utilizaram do método CI (Custo de Investimento), que resulta do custo total da planta (TPC) somado ao requerimento total de capital (TCR), para estimar o custo em função da capacidade nominal $(\$ / \mathrm{kW})$. Na Tabela 2 apresenta-se o custo da energia elétrica por $\mathrm{kW}$, através dos dados obtidos da EIA (2010, p. 7) e Ortiz (2011, p. 110), com base no método CI. 
TABELA 2 - CUSTO DA ENERGIA ELÉTRICA ATRAVÉS DE IGCC POR KW

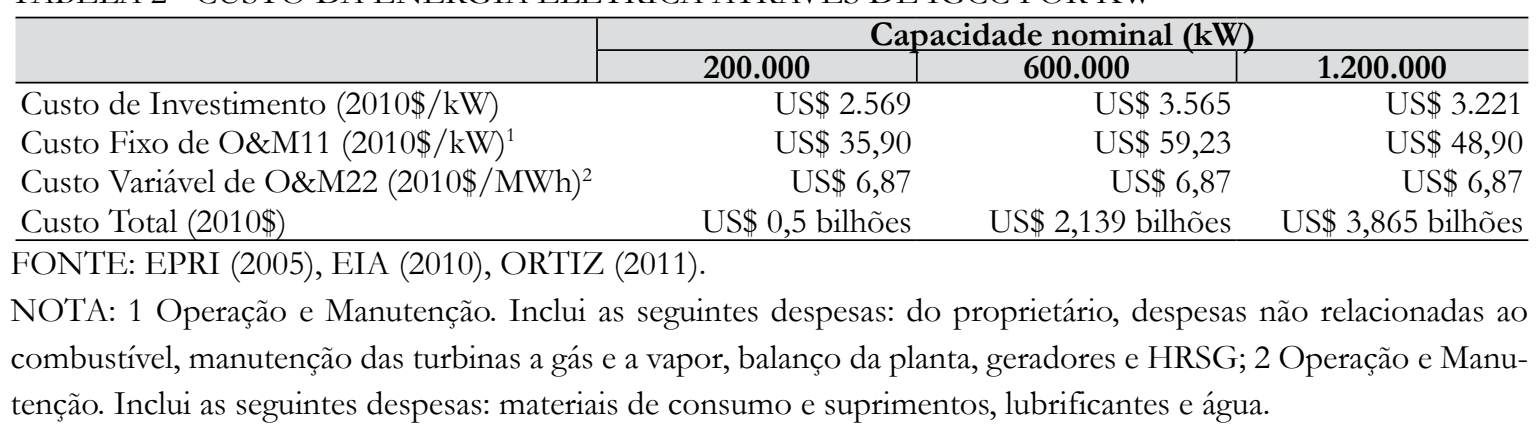

Segundo HOFFMAN (2010), o CI, que inclui os custos que incidem sobre a instalação de uma planta com tecnologia IGCC, geralmente expressado como o custo total por capacidade nominal, é ideal para realizar a comparação com os custos de plantas de diferentes tamanhos. No TPC está incluído o custo de instalação da planta, como equipamentos do processo, mão de obra direta e indireta, design, construção e gerência do projeto. O TCR inclui o TPC, o valor do dinheiro no tempo, os custos de início de operação e do proprietário.

Plantas de IGCC de pequeno porte são construídas com a finalidade de demonstração do funcionamento da tecnologia. Quando planejadas para comercialização, tornam-se extremamente inviáveis. Segundo a EPRI (2005, p. 26), “Os custos atrelados a uma planta de 2 MW ficariam em torno de 32,3 milhões de dólares". Entretanto plantas de maior capacidade se encontram em funcionamento comercial.

\subsection{CUSTOS DE GERAÇÃO DE ENERGIA ELÉTRICA ATRAVÉS DE FONTE EÓLI- CA E SOLAR}

Há um crescente interesse no desenvolvimento de energias renováveis provenientes das tecnologias solar e eólica, devido aos benefícios ambientais e de segurança energética que essas proporcionam, fato que torna a energia renovável atraente para os investidores. Apesar desses e outros benefícios, problemas relacionados aos custos têm impedido o desenvolvimento desta matriz em larga escala (NATIONAL RENEWABLE ENERGY LABORATORY, 2008).

A utilização do potencial da radiação solar e energia cinética dos ventos, como matriz energética, é uma opção capaz de auxiliar no fornecimento de energia elétrica com baixo índice de carbono para economias mundias. Porém, seria necessário realizar transformações em todo o sistema elétrico para explorar amplamente as fontes de energia renovável disponíveis. A geração de eletricidade por meio solar e/ou eólico são tecnologias que disputam espaço no mercado energético mundial, assim como a tecnologia IGCC. A finalidade de tais tecnologias 
é a geração sem a emissão de gases poluentes emitidos pelas unidades térmicas convencionais. Como forma de evidenciar a diferença do custo relacionado na geração do kW existente entre as tecnologias renováveis abordadas neste artigo, elaborou-se as Tabelas 3 e 4, para posterior comparação dos custos associadas a estas tecnologias, onde os custos fixos de operação e manutenção são referentes as despesas do proprietário, manutenção e possível substituição nos equipamentos auxiliares, no gerador e turbina.

TABELA 3 - CUSTO DA ENERGIA ELÉTRICA EÓLICA POR KW

\begin{tabular}{l|rrr}
\hline & \multicolumn{3}{|c}{ Capacidade nominal $(\mathbf{k W})$} \\
\cline { 2 - 4 } & $\mathbf{5 0 . 0 0 0}$ & $\mathbf{1 0 0 . 0 0 0}$ & \multicolumn{1}{c}{$\mathbf{2 0 0 . 0 0 0}$} \\
\hline Custo de Investimento $(2010 \$ / \mathrm{kW})$ & US $\$ 1.896$ & US $\$ 2.438$ & US $\$ 2.313$ \\
Custo Fixo de O\&M $(2010 \$ / \mathrm{kW})$ & US $\$ 30,92$ & US $\$ 28,07$ & US $\$ 25,33$ \\
Custo Variável de O\&M $(2010 \$ / \mathrm{MWh})$ & US $\$ 0,00$ & US $\$ 0,00$ & US $\$ 0,00$ \\
Custo Total $(2010 \$)$ & US $\$ 94,8$ milhões & US $\$ 243,8$ milhões & US $\$ 462,6$ milhões \\
\hline
\end{tabular}

FONTE: EIA (2010).

TABELA 4 - CUSTO DA ENERGIA ELÉTRICA SOLAR POR KW

\begin{tabular}{|c|c|c|c|}
\hline & \multicolumn{3}{|c|}{ Capacidade nominal (kW) } \\
\hline & 500 & 7.000 & 150.000 \\
\hline Custo de Investimento $(2010 \$ / \mathrm{kW})$ & US\$ 8.030 & US\$ 6.050 & $\mathrm{US} \$ 4.755$ \\
\hline Custo Fixo de O\&M33 $(2010 \$ / \mathrm{kW})^{3}$ & US\$ 31,12 & US\$26,04 & US\$ 16,70 \\
\hline Custo Variável de O\&M (2010\$/MWh) & US\$ 0,00 & US\$ 0,00 & $\mathrm{US} \$ 0,00$ \\
\hline Custo Total $(2010 \$)$ & US\$ 4,015 milhões & US\$ 42,35 milhões & US\$713,2 milhões \\
\hline
\end{tabular}

FONTE: EIA (2010).

NOTA: 3 Operação e Manutenção. Inclui as seguintes despesas: do proprietário, manutenção periódica do inversor e limpeza periódica do painel.

\section{ANÁLISE E DISCUSSÃO DOS RESULTADOS}

Tendo como premissa a questão ambiental, a implantação de uma unidade IGCC apresenta como ponto positivo a sua aptidão para adição de sistemas de CCS (Carbon Capture and Storage - captura e armazenamento de carbono), proporcionando outras vantagens como baixas emissões de compostos de enxofre e mercúrio, além de maior flexibilidade em relação a insumos e produtos de processo. Deste modo, temos dois produtos para comercialização: energia elétrica e os créditos de carbono.

Quando comparada com energia solar e eólica (ver Tabela 5) a tecnologia IGCC demonstrou ser competitiva somente em relação à energia solar, que, apesar de não possuir custo variável de operação e manutenção, possui alto custo de investimento.

Mesmo possuindo um custo de investimento similar ao da energia eólica e abaixo da energia solar, o custo de manutenção e operação aumenta significativamente as despesas de uma planta IGCC. Apesar desse fato, o custo nivelado de eletricidade em IGCC demonstra ser menor quando comparado ao solar.

Buscando comparar os custos relacionados à implantação de unidades de geração de 
energia elétrica por meio de eólica, solar e IGCC, confeccionou-se a Tabela 5, com o intuito de verificar qual tecnologia apresenta menor custo, tendo a mesma capacidade, i.e., média de 200 MW.

TABELA 5 - COMPARAÇÃO ENTRE TECNOLOGIAS

\begin{tabular}{|c|c|c|c|}
\hline & \multicolumn{3}{|c|}{ Tipo de tecnologia } \\
\hline & IGCC (200 MW) & Eólica (200 MW) & Solar (150 MW) \\
\hline Custo de Investimento $(2010 \$ / \mathrm{kW})$ & US\$2.569 & US $\$ 2.313$ & US\$ 4.755 \\
\hline Custo Fixo de O\&M (2010\$/kW) & US\$ 35,90 & US\$25,33 & US $\$ 16,70$ \\
\hline Custo Variável de O\&M (2010\$/MWh) & US\$ 6,87 & US $\$ 0,00$ & US\$ 0,00 \\
\hline Custo Total $(2010 \$)$ & US\$ 513,8 milhões & US\$ 462,6 milhões & US\$ 713,2 milhões \\
\hline
\end{tabular}

FONTE: EIA (2010).

Segundo Lazard (2009), a tecnologia que apresenta o menor custo nivelado de eletricidade (\$/MWh) é a eólica, seguida pelo IGCC e por último a solar, que apresentam seus custos na faixa de 113,149 e 182 dólares, respectivamente, dado este que comprova o atrativo da geração de eletricidade através da tecnologia eólica, mas que também mostra que a tecnologia IGCC possui total condição de, num curto prazo, ser viável economicamente.

\section{CONCLUSÕES}

A tecnologia IGCC apresenta seu custo de investimento abaixo das outras tecnologias, haja vista a capacidade de gerar energia elétrica através do carvão mineral, atendendo às exigências ambientais vigentes. Entretanto, essa tecnologia não demonstra ser viável, quando comparada com a geração de energia elétrica através da energia solar e eólica, devido ao fato de possuir um elevado custo de manutenção e operação.

Apesar das vantagens apresentadas relacionadas à implantação de uma planta IGCC, as mesmas ainda não se estabeleceram em grande escala no mercado, sendo que seus obstáculos são o alto custo da tecnologia, menor confiabilidade, longo tempo de construção e a falta de experiência de operação.

A fim de ampliar a utilização desta tecnologia, faz-se necessária a sua integração com a captura do carbono que é coletado antes da combustão, ou seja, o Ciclo Combinado poderá ser considerado tecnicamente praticável. Como a introdução desta tecnologia em grande escala no setor elétrico depende da sua viabilidade econômica, justifica-se seu maior retorno quando comparada com as tecnologias de geração de eletricidade.

\section{REFERÊNCIAS}

DOLEZAL, R. Kombinierte gas-und dampfkraftwerke. 1. ed. Berlin Heidelberg: Springerverlag, 2001. 
EIA. Energy Information Administration. Estados Unidos da América, 2010. Disponível em: <http://www.eia.gov/oiaf/beck_plantcosts/pdf/updatedplantcosts.pdf>. Acesso em: $29 / 5 / 2011$.

ELETRIC POWER RESEARCH INSTITUTE, Gaseification Technology Status. Estados Unidos da América, 2005. Disponível em: <http://my.epri.com/portal/server.pt?>. Acesso em: 29/5/2011.

HOFFMANN, B. S. O ciclo combinado com gaseificação integrada e captura de $\mathbf{C O}_{2}$ : uma solução para mitigar as emissões de $\mathrm{CO}_{2}$ em termelétricas a carvão em larga escala no curto prazo? Dissertação (Mestrado em Planejamento Energético), COPPE, Rio de Janeiro, 2010.

LAZARD. Estados Unidos da América, 2009. Disponível em: < http:/ /efile. mpsc.state. mi.us/ efile/docs/15996/0145.pdf>. Acesso em: 5/7/2011.

MME - Ministério de Minas e Energia, e Empresa de Pesquisa Energética - EPE. Balanço Energético Nacional - ano base 2009. Brasília, 2010.

NATIONAL RENEWABLE ENERGY LABORATORY. Innovations in wind and solar PV financing. Estados Unidos da América 2008. Disponível em: <http://www.nrel.gov/docs/ fy08osti/ 42919.pdf>. Acesso em: 5/6/2011.

ORTIZ, P. A. S. Avaliação técnico-econômica de sistemas IGCC utilizando coque de petróleo e carvão mineral como combustível. 141 p. Dissertação (Mestrado em Engenharia Mecânica), Universidade Federal de Itajubá, 2011. Disponível em: <http://adm-net-a.unifei. edu.br/phl/pdf/0038056.pdf>. Acesso em: 31/5/2011.

REGO, E. E. Avaliação da viabilidade de um empreendimento de geração de energia hidrelétrica. 129 p. Monografia (Bacharel em Ciências Econômicas), Universidade de São Paulo, São Paulo, 2004.

WORLD COAL ASSOCIATION. Disponível em: <http://thefraserdomain.typepad.com/ energy/2005/09/about_igcc_powe.html >. Acesso em: 1/6/2011. 
